\title{
ARTICLE
}

Epidemiology

\section{Type 2 diabetes and risk of colorectal cancer in two large U.S. prospective cohorts}

Yanan Ma ${ }^{1,2}$, Wanshui Yang ${ }^{2,3}$, Mingyang Song ${ }^{4,5,6}$, Stephanie A. Smith-Warner ${ }^{4,7}$, Juhong Yang ${ }^{8}$, Yanping $\mathrm{Li}^{4}$, Wenjie Ma ${ }^{9}$, Yang Hu ${ }^{4,7}$, Shuji Ogino ${ }^{9,10,11,12}$, Frank B. Hu ${ }^{2,4}$, Deliang Wen ${ }^{1}$, Andrew T. Chan ${ }^{2,5,6,11,13}$, Edward L. Giovannucci ${ }^{2,4,9}$ and Xuehong Zhang $^{2}$

BACKGROUND: Previous studies have shown a positive association between type 2 diabetes (T2D) and colorectal cancer (CRC) risk. However, it is uncertain whether this association differs by duration of T2D or sex. We thus investigated the associations of T2D and its duration with the risk of incident CRC.

METHODS: We followed 87,523 women from the Nurses' Health Study (1980-2012) and 47,240 men from the Health Professionals Follow-up Study (1986-2012). Data on physician-diagnosed T2D was collected at baseline with a questionnaire and updated biennially. Cox regression models were used to estimate multivariable-adjusted hazard ratios (HRs) and 95\% confidence intervals (Cls).

RESULTS: We documented 3000 CRC cases during up to 32 years of follow-up. Among men, T2D was associated with increased risk of CRC compared to those without T2D (HR: 1.42; 95\% Cl: 1.12-1.81). This positive association persisted in sensitivity analyses by excluding CRC identified within 1 year of diabetes diagnosis and patients with T2D who used hypoglycaemic medications. Among women, T2D was positively, but not statistically significantly, associated with CRC risk (HR: 1.17; 95\% Cl: 0.98-1.39).

CONCLUSIONS: Our findings support that T2D was associated with a moderately higher risk of developing CRC in men; a weaker, nonsignificant positive association was observed in women.

British Journal of Cancer (2018) 119:1436-1442; https://doi.org/10.1038/s41416-018-0314-4

\section{INTRODUCTION}

Colorectal cancer is the third most commonly diagnosed cancer in the US ${ }^{1}$ and worldwide. ${ }^{2}$ A number of epidemiological studies have shown that individuals with type 2 diabetes have a higher risk of developing colorectal cancer compared with their nondiabetic counterparts. ${ }^{3-22}$ Elevated insulin due to insulin resistance and hyperinsulinaemia, which occurs in the early stages of type 2 diabetes, ${ }^{23}$ has been hypothesised to partially explain the proposed type 2 diabetes-colorectal cancer association. ${ }^{24}$ Insulin is an important growth factor of colonic epithelial cells and is a mitogen of tumour cell growth in vitro. ${ }^{24}$ Insulin may also promote colorectal carcinogenesis indirectly through increasing bioavailable insulin-like growth factor 1, which could enhance cell proliferation and inhibit apoptosis. ${ }^{24}$ Another potential biological link between type 2 diabetes and cancer is hyperglycaemia, which may impair the effect of ascorbic acid on intracellular metabolism, reduce the effectiveness of the immune system, and regulate the level of reactive oxygen species, which could favour cancer development and progression in diabetic patients. ${ }^{25}$

Several issues regarding the association between type 2 diabetes and colorectal cancer risk remained unresolved. First, the relationship between duration of diabetes and colorectal cancer risk remains uncertain. Some studies have reported a higher risk of colorectal cancer with prolonged duration of type 2 diabetes ${ }^{3,5,26}$ or with short duration, ${ }^{13}$ whereas other studies have shown no difference in risk by duration of type 2 diabetes., ${ }^{72}$ Second, most previous studies only examined a single measurement of diabetes at baseline, ${ }^{4,6,8,10,14,15}$ which cannot account for new diagnoses of diabetes during follow-up. Last, published data has shown inconclusive results about the association of type 2 diabetes with the risk of different subsites of colorectal cancer. ${ }^{27-29}$ The potential gender difference in the type 2 diabetes and colorectal cancer association also remains unclear. This issue is of interest because sex hormones (e.g. testosterone, oestrogen and progesterone) may play a role in colorectal cancer. ${ }^{30-35}$

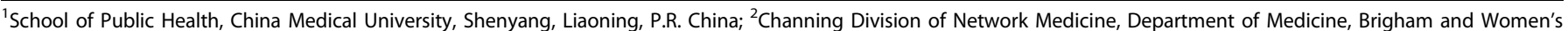

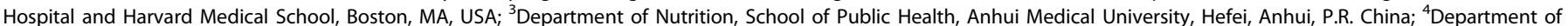

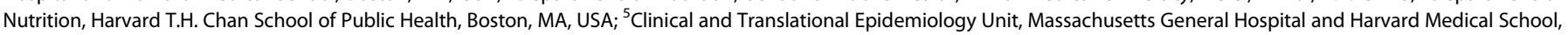

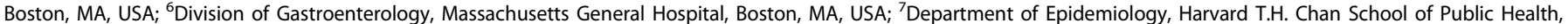

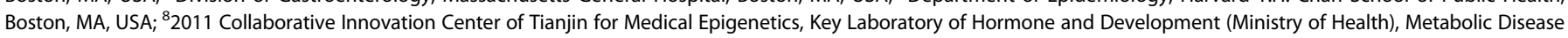

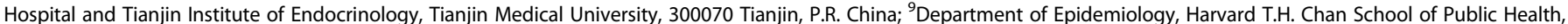

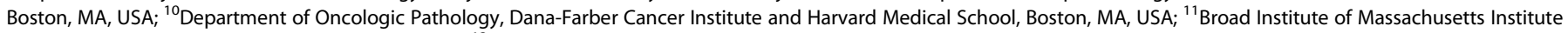

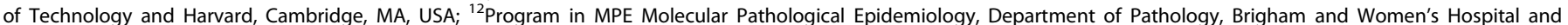
Harvard Medical School, Boston, MA, USA and ${ }^{13}$ Department of Immunology and Infectious Diseases, Harvard T.H. Chan School of Public Health, Boston, MA, USA Correspondence: Xuehong Zhang (xuehong.zhang@channing.harvard.edu)
}

Received: 5 May 2018 Revised: 27 September 2018 Accepted: 3 October 2018

Published online: 7 November 2018 
To address these issues, we examined whether physiciandiagnosed type 2 diabetes and its duration are associated with the risk of colorectal cancer in the Nurses' Health Study and the Health Professionals Follow-up Study. In addition, we also examined potential differences in the association by sex.

\section{METHODS}

Study population

We conducted this study by using data from the two large-scale prospective cohort studies: the Nurses' Health Study and the Health Professionals Follow-up Study. In brief, the Nurses' Health Study was initiated in 1976 and included a total of 121,700 US female registered nurses aged 30-55 years. The Health Professionals Follow-up Study was established in 1986 and included 51,529 male health professionals aged 40-75 years. Participants were followed up biennially by validated questionnaires on demographics, medical conditions, and health-related behaviours. The validity of questionnaire has been described in detail previously. ${ }^{36}$ The follow-up rates have been $>90 \%$ in each cohort.

For the current study, we used 1980 as the baseline for the Nurses' Health Study and 1986 for the Health Professionals Followup Study. We excluded participants with missing data on diabetes, with unconfirmed diabetes, and with a history of cancer (except for non-melanoma skin cancer) or inflammatory bowel disease at baseline. After exclusions, the final study population consisted of 87,523 women from the Nurses' Health Study and 47,240 men from the Health Professionals Follow-up Study. The study protocol was approved by the institutional review board of the Brigham and Women's Hospital and the Human Subjects Committee Review Board of the Harvard T. H. Chan School of Public Health.

Assessment of type 2 diabetes

In the baseline and all biennial questionnaires, the participants were asked if and when they had ever been diagnosed with type 2 diabetes by a physician. To confirm the self-reported cases of physician-diagnosed diabetes, a supplementary questionnaire was then sent for ascertainment to obtain details about the date of diagnosis, symptoms, diagnostic tests, and hypoglycaemic treatment. Beginning in 1986 in each cohort, supplementary questionnaires were administered to collect information on antidiabetic drug use (insulin or hypoglycaemic agent) as described in detail previously. ${ }^{37}$ Cases of type 2 diabetes were defined as initially self-reported diabetes and subsequently confirmed by the validated questionnaires. ${ }^{38,39}$ Consistent with previous studies from the same cohorts, ${ }^{40}$ confirmed diabetes requires at least one of the following criteria reported on the supplementary questionnaire according to the American Diabetes Association: one or more classic symptoms (excessive thirst, polyuria or frequent urination, weight loss, hunger) plus fasting plasma glucose $\geq 7.0 \mathrm{mmol} / \mathrm{l}$ or random plasma glucose levels $\geq 11.1 \mathrm{mmol} / \mathrm{l} ; \geq 2$ elevated plasma glucose concentrations on different occasions (fasting glucose $\geq 7.0 \mathrm{mmol} / \mathrm{l}$, random plasma glucose $\geq 11.1 \mathrm{mmol} / \mathrm{l}$, and/or plasma glucose $\geq 11.1 \mathrm{mmol} / \mathrm{l}$ after $\geq 2 \mathrm{~h}$ shown by oral glucose tolerance testing) in the absence of symptoms; or treatment with hypoglycaemic medication. Before 1998 , fasting plasma glucose of $7.8 \mathrm{mmol} / \mathrm{l}$ was considered for the diagnosis of diabetes according to the National Diabetes Data Group criteria. The validity of the supplementary questionnaire has been confirmed through medical records. In each cohort, diagnosis of type 2 diabetes was confirmed in $>97 \%$ of the cases. ${ }^{39,41}$

\section{Assessment of covariates}

Information on body weight, physical activity, other lifestyle information, and medical history was obtained at baseline and was updated through biennial questionnaires. Height was ascertained in 1980 in the Nurses' Health Study and in 1986 in the Health Professionals Follow-up Study. We calculated body mass index (BMI) as weight in kilograms divided by height in metres squared. Early-life BMI was measured at 18 years in the Nurses' Health Study and at 21 years in the Health Professionals Follow-up Study. Each cohort administered food-frequency questionnaires ( 130 food items) to obtain information on usual dietary intake over the previous year at baseline and repeated almost every 4 years thereafter, which allowed us to calculate intakes of nutrients and foods such as alcohol, calories, calcium, dietary fibre, sugar, carbohydrates, folate, vitamin D, processed meat, and red meat. ${ }^{42-46}$

\section{Identification of colorectal cancer cases}

In both cohorts, we identified cases of colorectal cancer through biennial questionnaires. ${ }^{47}$ All possible cancer cases were confirmed through review of medical and pathological records. The incident colorectal cancer cases were defined as a primary tumour with International Classification of Diseases-9 codes of 153 and 154. A study physician who was blinded to exposure data abstracted information on tumour anatomic location, stage, and histology. We searched state vital statistics records and the National Death Index to identify additional unreported cancer deaths. In this analysis, we considered both overall colorectal cancer and anatomic subsites, including colon cancer, proximal colon cancer (caecum, ascending colon, and transverse colon), distal colon cancer (descending colon and sigmoid colon), and rectal cancer (including recto-sigmoid).

\section{Statistical analyses}

Person-time for participants was calculated from the return of the baseline questionnaires until the date of colorectal cancer diagnosis, death, loss to follow-up, or the end of the follow-up (June 2012 for the Nurses' Health Study and January 2012 for the Health Professionals Follow-up Study), whichever came first. Consistent with our previous report, ${ }^{7}$ we evaluated the association with status (no versus yes) and duration of type 2 diabetes (0.1-5, $5.1-10,10.1-15$, and $>15$ years) within each cohort. We used Cox regression models to calculate the multivariable hazard ratios (HRs) and $95 \%$ confidence intervals $(95 \% \mathrm{Cls})$. Age in months and calendar year at the start of follow-up of each 2-year questionnaire cycle were used as stratification variables in the model. In the multivariable analyses, we adjusted for race (Whites or non-Whites); family history of diabetes (yes or no); regular aspirin use (yes or no); BMI $\left(<25,25-<27.5,27.5-<30, \geq 30 \mathrm{~kg} / \mathrm{m}^{2}\right)$; history of colorectal cancer in a parent or sibling (yes or no); history of endoscopy/ sigmoidoscopy (yes or no); smoking habits $(0,1-10$, or $>10$ pack-years); alcohol consumption $(<5,5-<15, \geq 15 \mathrm{~g} /$ day); physical activity $(<3,3-<27, \geq 27$ METS-hours/week); total calories (tertiles); energy-adjusted intake of total folate; total calcium; total vitamin D; processed meat; and beef, pork, or lamb as a main dish (all in tertiles). For women, we additionally adjusted for postmenopausal hormone use (premenopausal, never, past, or current user). Confounders were updated over the follow-up periods to reduce random within-person measurement error. Test of statistical heterogeneity in the association of type 2 diabetes and colorectal cancer risk by gender was assessed by the $\mathrm{Q}$ statistics. ${ }^{48}$ For the two cohorts, there were about $0.1-3 \%$ missing data in processed meat, beef, pork, or lamb as the main dish or for family history of colorectal cancer. Given the generally low proportion of missing data in these covariates, we have included the individuals with missing in family history of colorectal cancer in the no family history group and missing in intake of processed meat or beef, pork, or lamb as the main dish in the tertile 1 group.

Previous studies have suggested that smoking ${ }^{20}$ may modify the association between diabetes and colorectal cancer; and BMI, physical activity, and moderate alcohol consumption are associated with insulin resistance. ${ }^{49-51}$ Therefore, we considered these potential variables that might modify the diabetes and colorectal 
Type 2 diabetes and risk of colorectal cancer in two large U.S....

Y Ma et al.

Table 1. Age-standardised characteristics of participants according to status and duration of type 2 diabetes in the Nurses' Health Study (NHS) and Health Professionals Follow-up Study (HPFS)

\begin{tabular}{|c|c|c|c|c|c|c|}
\hline & No diabetes & Diabetes & $0.1-5$ years & $5.1-10$ years & $10.1-15$ years & $>15$ years \\
\hline Age (year) ${ }^{\mathrm{b}}$ & $60.2(11.3)$ & $67.7(9.4)$ & $65.6(9.3)$ & $67.5(9.3)$ & $68.5(9.4)$ & $70.0(9.2)$ \\
\hline Whites, \% & 97.6 & 95.6 & 95.9 & 95.4 & 95.6 & 95.7 \\
\hline Body mass index, $\mathrm{kg} / \mathrm{m}^{2}$ & $24.7(4.2)$ & $30.2(5.7)$ & $30.1(5.5)$ & $30.4(5.7)$ & $30.4(5.9)$ & $29.4(5.9)$ \\
\hline Smoking, pack years & $12.9(19.0)$ & $13.8(20.0)$ & $14.2(20.2)$ & $13.9(20.1)$ & $13.8(20.2)$ & $12.9(19.8)$ \\
\hline Regular aspirin use, $\%$ & 39.9 & 47.3 & 44.9 & 46.2 & 49.3 & 50.9 \\
\hline History of endoscopy/sigmoidoscopy, \% & 26.1 & 26.6 & 27.4 & 26.8 & 25.4 & 26.5 \\
\hline Family history of colorectal cancer, \% & 12.2 & 12.6 & 12.6 & 12.8 & 12.4 & 12.9 \\
\hline Family history of diabetes, $\%$ & 26.6 & 55.9 & 50.7 & 54.8 & 58.5 & 63.7 \\
\hline Total calcium, g/day & 933 (359) & $912(338)$ & $911(340)$ & 910 (339) & $907(335)$ & $929(340)$ \\
\hline Total fibre, g/day & $17.5(4.9)$ & $17.6(4.7)$ & $17.3(4.6)$ & $17.5(4.7)$ & $17.7(4.6)$ & $18.0(4.7)$ \\
\hline Total sugar, g/day & $94.3(26.7)$ & $90.5(26.1)$ & $93.0(26.4)$ & $90.4(25.8)$ & $88.0(25.5)$ & $88.1(26.0)$ \\
\hline Total carbohydrates, g/day & $181(32)$ & $179(30)$ & $181(30)$ & $179(31)$ & $178(30)$ & $178(30)$ \\
\hline Total folate, mcg/day & $424(213)$ & $424(198)$ & 417 (199) & $423(201)$ & $424(191)$ & $442(195)$ \\
\hline Vitamin D intake (IU/day) & $356(224)$ & $358(210)$ & $351(212)$ & $358(214)$ & $360(205)$ & $376(204)$ \\
\hline Processed meat, servings/week & $0.94(1.26)$ & $1.19(1.45)$ & $1.15(1.44)$ & $1.20(1.47)$ & $1.23(1.48)$ & $1.19(1.38)$ \\
\hline Beef/pork/lamb as the main dish, servings/week & $2.07(1.43)$ & $2.24(1.46)$ & $2.21(1.43)$ & $2.27(1.52)$ & $2.28(1.47)$ & $2.24(1.42)$ \\
\hline \multicolumn{7}{|l|}{ Men (HPFS) } \\
\hline Age $(\text { year })^{\mathrm{b}}$ & $63.8(11.2)$ & $70.7(9.1)$ & $68.2(9.1)$ & $71.4(8.7)$ & $73.4(8.2)$ & $76.2(8.0)$ \\
\hline History of endoscopy/sigmoidoscopy, \% & 53.5 & 60.7 & 59.0 & 62.5 & 68.8 & 61.6 \\
\hline Family history of colorectal cancer, $\%$ & 12.2 & 11.4 & 11.5 & 11.5 & 11.9 & 10.7 \\
\hline Family history of diabetes, $\%$ & 25.0 & 47.5 & 46.9 & 46.8 & 47.6 & 38.1 \\
\hline Total calorie intake, kcal/day & $1977(555)$ & $1991(564)$ & $1995(564)$ & $1992(544)$ & $1965(503)$ & $1815(526)$ \\
\hline Alcohol, g/day & $11.1(13.9)$ & $8.5(12.2)$ & $8.8(12.4)$ & $8.6(12.3)$ & $7.8(10.8)$ & $12.1(13.7)$ \\
\hline Total calcium, g/day & $935(373)$ & $936(354)$ & $930(357)$ & $949(344)$ & 959 (324) & $927(362)$ \\
\hline Total fibre, g/day & $22.1(6.6)$ & $21.9(6.0)$ & $21.6(6.0)$ & $22.2(5.9)$ & $22.6(5.8)$ & $21.5(6.4)$ \\
\hline Total sugar, g/day & $110.5(30.4)$ & $104.1(27.3)$ & $106.5(27.7)$ & $102.8(26.2)$ & $99.6(23.4)$ & $89.3(29.4)$ \\
\hline Total carbohydrates, g/day & $243(39)$ & $237(34)$ & $238(35)$ & $237(33)$ & $236(32)$ & $226(36)$ \\
\hline Total folate, mcg/day & $542(254)$ & $545(231)$ & $534(232)$ & $558(222)$ & $579(208)$ & $572(218)$ \\
\hline Vitamin D intake (IU/day) & $434(267)$ & $424(243)$ & $422(247)$ & $431(233)$ & $430(212)$ & $463(201)$ \\
\hline Processed meat, servings/week & $1.08(1.49)$ & $1.31(1.45)$ & $1.27(1.45)$ & $1.33(1.54)$ & $1.33(1.31)$ & $1.54(1.30)$ \\
\hline Beef/pork/lamb as the main dish, servings/week & $1.72(1.40)$ & $1.96(1.42)$ & $1.95(1.44)$ & $1.94(1.29)$ & $1.94(1.25)$ & $2.05(1.15)$ \\
\hline
\end{tabular}


Type 2 diabetes and risk of colorectal cancer in two large U.S...

Y Ma et al.

Table 2. Status and duration of type 2 diabetes and colorectal cancer risk in the Nurses' Health Study (NHS) and Health Professionals Follow-up Study (HPFS)

\begin{tabular}{|c|c|c|c|c|}
\hline & \multicolumn{2}{|c|}{ Women $(\mathrm{NHS}, N=1790)$} & \multicolumn{2}{|c|}{ Men (HPFS, $N=1210)$} \\
\hline No diabetes & 1631 & 1 (Reference) & 1129 & 1 (Reference) \\
\hline \multicolumn{5}{|l|}{ Diabetes } \\
\hline Age-adjusted model & 159 & $1.32(1.12,1.55)$ & 81 & $1.57(1.24,1.98)$ \\
\hline Multivariable-adjusted model $^{a}$ & 159 & $1.17(0.98,1.39)$ & 81 & $1.42(1.12,1.81)$ \\
\hline Multivariable-adjusted model $^{\mathrm{a}}$ & 51 & $1.16(0.87,1.54)$ & 37 & $1.39(0.99,1.95)$ \\
\hline \multicolumn{5}{|l|}{$5.1-10$ years } \\
\hline Age-adjusted model & 42 & $1.38(1.01,1.88)$ & 25 & $1.59(1.06,2.38)$ \\
\hline Multivariable-adjusted model $^{\mathrm{a}}$ & 42 & $1.20(0.88,1.64)$ & 25 & $1.42(0.94,2.15)$ \\
\hline \multicolumn{5}{|l|}{$10.1-15$ years } \\
\hline Age-adjusted model & 42 & $1.37(1.00,1.87)$ & 5 & $1.59(0.65,3.89)$ \\
\hline Multivariable-adjusted model $^{a}$ & 42 & $1.26(0.91,1.72)$ & 5 & $1.37(0.56,3.38)$ \\
\hline \multicolumn{5}{|c|}{$\begin{array}{l}\text { adjusted for age; race (Whites or non-Whites); family history of diabetes (yes or no); regular aspirin use (yes or no); } \mathrm{BMI}\left(<25,25-<27.5,27.5-<30, \geq 30 \mathrm{~kg} / \mathrm{m}^{2}\right) \text {; } \\
\text { history of colorectal cancer in a parent or sibling (yes or no); history of endoscopy/sigmoidoscopy (yes or no); smoking }(0,0-<10, \geq 10 \mathrm{pack}-\mathrm{years}) ; \text { alcohol } \\
\text { consumption }(<5,5-<15, \geq 15 \mathrm{~g} / \text { day); physical activity }(<3,3-<27, \geq 27 \mathrm{METS} \text {-hours/week); total calorie intake (tertiles); energy-adjusted intake of total folate; } \\
\text { total calcium; total vitamin } \mathrm{D} \text { intake; processed meats; and beef, pork, or lamb as a main dish (all in tertiles). We also adjusted for postmenopausal hormone } \\
\text { use (premenopausal, never, past, or current user) in women. Bold text indicated a statistically significant association }\end{array}$} \\
\hline
\end{tabular}

cancer risk associations. We conducted subgroup analyses by smoking status (never smoker versus ever smoker), BMI $(\geq 25$ versus $<25 \mathrm{~kg} / \mathrm{m}^{2}$ ), early life BMl ( $\geq 23$ versus $\left.<23 \mathrm{~kg} / \mathrm{m}^{2}\right)$, physical activity ( $<9$ versus $\geq 9$ METS-hours/week), alcohol drinking (nondrinkers versus drinkers), and other selected risk factors including age ( $\geq 65$ versus $<65$ years) and family history of diabetes (yes versus no). We used Wald test to examine whether the crossproduct terms between these variables and diabetes status were statistically significant.

We conducted several secondary analyses. First, we evaluated whether the associations between diabetes (status and duration) and colorectal cancer risk differed by anatomic subsites (colon cancer, proximal colon cancer, distal colon cancer, and rectal cancer). ${ }^{28}$ Second, we restricted analyses to untreated diabetes by excluding patients with type 2 diabetes who have used hypoglycaemic medication (insulin or oral hypoglycaemic agent). A total of 195 cases (146 for women and 49 for men) were excluded from our analysis to investigate whether diabetes treatments affect the association between type 2 diabetes and colorectal cancer risk. Last, we excluded colorectal cancer cases diagnosed within 1 year of diabetes diagnosis to minimise the potential for reverse causality and detection bias.

All statistical analyses were conducted using the SAS software (Version 9.3; SAS Institute, Cary, NC, USA). All statistical tests were two sided, and the significance level was set at $P<0.05$.

\section{RESULTS}

During 3,547,837 person-years of follow-up, we documented a total of 3000 incident cases of colorectal cancer (1790 in women and 1210 in men). In both cohorts, participants with type 2 diabetes were older and more likely to smoke and have a family history of diabetes (Table 1). Moreover, participants with type 2 diabetes were more likely to have higher BMI and use aspirin and were less likely to be physically active, drink alcohol, and consume sugar. Patients with longer duration of type 2 diabetes generally tended to be more physically active and consume less sugar and carbohydrates but were more likely to consume processed meats (especially in men).

Among men, type 2 diabetes was associated with increased risk of incident colorectal cancer (multivariable HR: $1.42 ; 95 \% \mathrm{Cl}$ : 1.12-1.81) (Table 2). The positive association for men persisted in sensitivity analyses excluding colorectal cancer cases diagnosed within 1 year of type 2 diabetes diagnosis (multivariable HR: 1.40; $95 \% \mathrm{Cl}$ : 1.10-1.78). Additionally, we observed similar positive associations for each tumour anatomic subsite (Supplementary Table 1) and when we restricted cases to only those with untreated diabetes (Supplementary Table 2). Among women, type 2 diabetes showed only a modest, borderline significant association with colorectal cancer risk (HR: $1.17 ; 95 \% \mathrm{Cl}$ : 0.98-1.39) (Table 2). The heterogeneity test did not achieve statistical significance ( $P=0.20$ for men versus women). Colorectal cancer risk did not vary with type 2 diabetes duration in either men or women.

In stratified analyses (Table 3), the association between type 2 diabetes and colorectal cancer risk appeared slightly stronger among ever smokers ( $P$-interaction $=0.04$ for women) and nondrinkers ( $P$-interaction $=0.05$ for men). Additionally, the association was also slightly stronger for those with higher BMI or early life BMI and individuals with a family history of diabetes, but none of the $P$-values were statistically significant.

\section{DISCUSSION}

In these two large prospective cohort studies, with $>26$ years of follow-up, we found that type 2 diabetes was associated with a moderately higher risk of incident colorectal cancer in men; a weaker, nonsignificant positive association was observed in 
Table 3. Stratified analyses of type 2 diabetes and risk of colorectal cancer in the Nurses' Health Study (NHS) and Health Professionals Follow-up Study (HPFS)

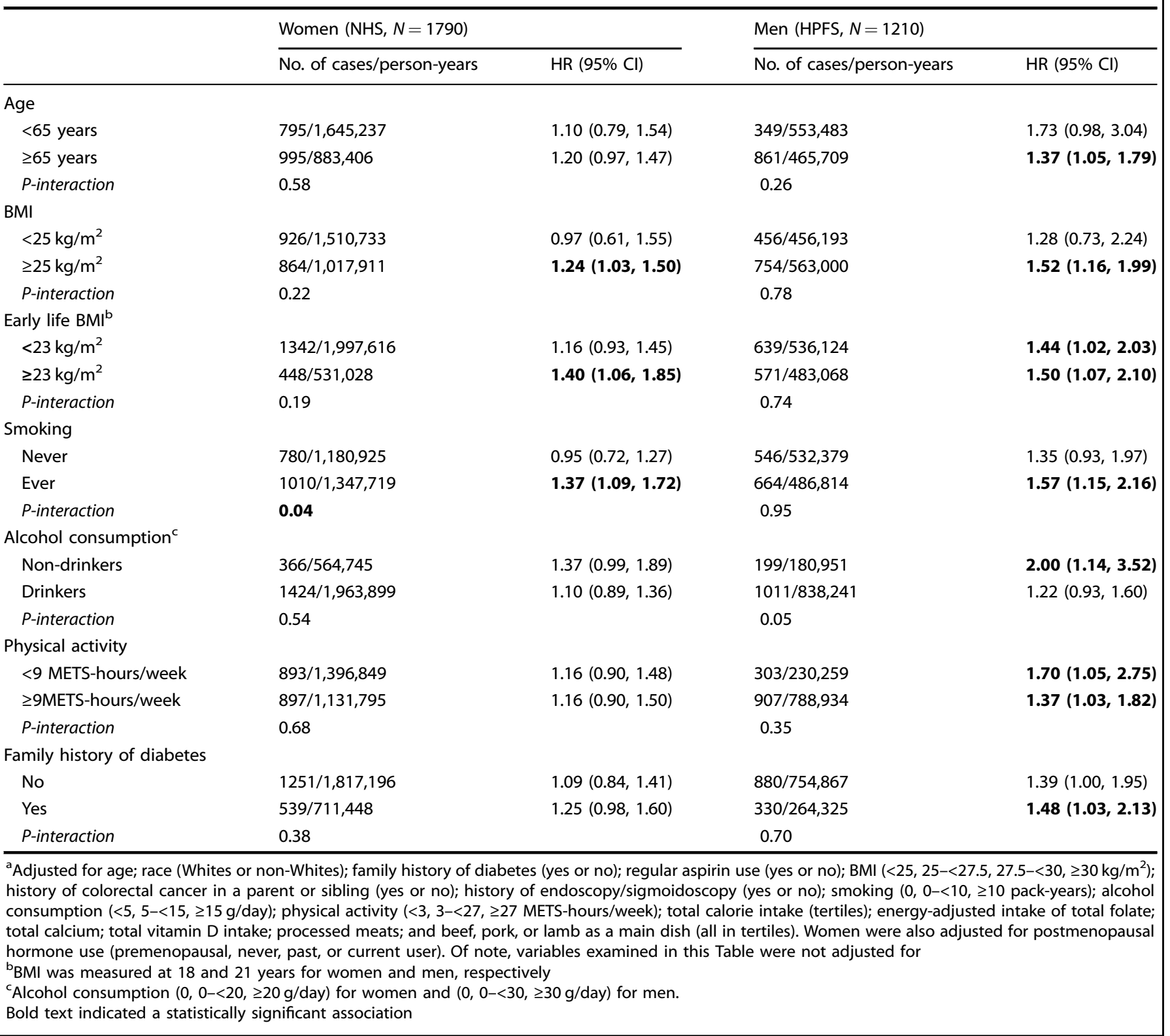

women. The positive association for men remained in sensitivity analyses that accounted for potential reverse causality by excluding colorectal cancer cases diagnosed within 1 year of diagnosis of type 2 diabetes and diabetes treatments and did not vary by diabetes duration.

In our previous study with follow-up through $1994,{ }^{7}$ we reported a significant association between type 2 diabetes and colorectal cancer risk among women in the Nurses' Health Study (HR: $1.43 ; 95 \% \mathrm{Cl}$ : 1.10-1.87). Positive associations of similar magnitude were reported for the women in the Cancer Prevention Study II $^{17}$ and the lowa Women's Health Study ${ }^{16}$ with follow-up through 1998 and 1999, respectively. In 2005, a meta-analysis of 15 studies (6 case-control and 9 cohort studies, including our prior Nurses' Health Study report) reported positive associations for diabetes and colorectal cancer risk of similar magnitude for men (HR: $1.29 ; 95 \% \mathrm{Cl}: 1.15-1.44$ ) and women (HR: 1.33; 95\% Cl: $1.23-1.44) .{ }^{9}$ However, most subsequent studies have reported a weaker positive ${ }^{14}$ or a null association ${ }^{3,18,20-22}$ between type 2 diabetes and colorectal cancer risk among women. This was consistent with our observation of a slightly weaker association in women but a moderately higher-risk association in men.

The possible differential association between women and men observed might be due to potential sex differences in the use of metformin and degree of glucose control. ${ }^{3}$ Compared with men, metformin has been more commonly used among women since the late 1990s. ${ }^{52}$ However, the potential effect of metformin use and colorectal cancer risk is not completely understood and more better designed studies are warranted to quantify the magnitude of the association between metformin use and colorectal cancer risk. Additionally, women generally tend to have better glucose control than men, even among those with type 2 diabetes. ${ }^{3}$ In fact, among individuals with type 2 diabetes, this difference is increasing. The National Health and Nutrition Examination Survey data showed that women with type 2 diabetes, but not men, have statistically significantly improved haemoglobin A1c (an indicator of glucose control) during the 
period from 1999 to 2004 in the US. ${ }^{53}$ Furthermore, differences in sex hormones may contribute to a more insulin-sensitive environment in women than in men. It may protect women from adverse outcomes related to hyperinsulinaemia. ${ }^{54,55}$ Observational studies $^{30-34,56}$ suggested an inverse association of endogenous oestrogens with the risk of colorectal cancer among women. Particularly, compared with women, men may have a higher insulin resistance partly due to the lack the protective effect of oestrogen..$^{54,55}$ Despite relatively comprehensive research on type 2 diabetes in relation to risk of colorectal cancer, ${ }^{29}$ current findings are inconsistent with regard to the potential gender difference. We observed a slightly stronger association in men than in women in this study but there was no statistically significant heterogeneity by gender. Nonetheless, the association between type 2 diabetes and colorectal cancer is strongly influenced by shared risk factors such as genetic variants, obesity, smoking, alcohol, and lack of physical activity. ${ }^{57,58}$ These potential factors might modify the diabetes and colorectal cancer risk differently in women and men, which requires further investigation.

We found that the type 2 diabetes-colorectal cancer association did not appear to be associated with diabetes duration. Because beta cells in the pancreas produce lower insulin levels as diabetes progresses into later stage, if hyperinsulinaemia plays a key role in this association, stronger positive associations would be expected in the early stage of diabetes. Thus our results did not support a strong role of hyperinsulinaemia in diabetes-colorectal cancer association.

We found slightly stronger associations between type 2 diabetes and colorectal cancer among ever smokers. This observation was consistent with a population-based, retrospective cohort study, which reported a stronger positive association among smokers. ${ }^{20}$ We also found a stronger association among non-drinkers of alcohol, indicating a potential interactive effect of alcohol consumption on type 2 diabetes. A similar, though not statistically significant, pattern was observed in women. Similar to that seen in cardiovascular disease, ${ }^{59}$ light-to-moderate intake of alcohol was associated with the reduced risk of colorectal cancer in some studies. ${ }^{60-62}$ Light-to-moderate alcohol consumption may increase insulin sensitivity and thus reduce insulin production. ${ }^{49,50}$ This was consistent with the observation that moderate alcohol consumption was associated with lower levels of plasma Cpeptide and lower risk of colorectal cancer. ${ }^{63}$ Thus the potential interaction between insulin and alcohol may protect patients with type 2 diabetes from serious outcomes of hyperinsulinaemia. However, the underlying mechanisms warrant further investigation.

Our present study has several strengths, including the prospective cohort design, high follow-up rates, validated colorectal cancer outcomes, and the use of repeated measures of physician-confirmed diabetes and lifestyle data. Our study also has several limitations. First, we have limited data on diabetes treatment. The observed association could be influenced by diabetes treatments because medications used to treat diabetes such as insulin injection ${ }^{64}$ or metformin use ${ }^{65}$ may affect the incidence of colorectal cancer. Moreover, we have limited data on glucose control because hyperglycaemia will also affect the cancer risk. However, the results were similar when we restricted the analysis among untreated diabetes (without any use of insulin or other hypoglycaemic agents). Second, our results might be influenced by detection bias, because newly diagnosed diabetes is more likely to be diagnosed with cancer due to increased detection around the time of the diagnosis of diabetes. ${ }^{66}$ However, we observed similar positive association when we excluded cases diagnosed within 1 year since type 2 diabetes.

In summary, our study suggests that type 2 diabetes was associated with a moderately higher risk of incident colorectal cancer in men; a weaker, nonsignificant association was observed in women. More studies are warranted to further investigate the underlying mechanisms. Our finding further underscores the importance of enhancing the effort to reduce the emerging epidemics of type 2 diabetes for colorectal cancer prevention and control.

\section{ACKNOWLEDGEMENTS}

We would like to thank the participants and staff of the Nurses' Health Study and the Health Professionals Follow-up Study for their valuable contributions as well as the following state cancer registries for their help: A.L., A.Z., A.R., C.A., C.O., C.T., D.E., F.L., G.A., I.D., I.L., I.N., I.A., K.Y., L.A., M.E., M.D., M.A., M.I., N.E., N.H., N.J., N.Y., N.C., N.D., O.H., O.K., O.R., P.A., R.I., S.C., T.N., T.X., V.A., W.A., W.Y.

\section{AUTHOR CONTRIBUTIONS}

Y.M. wrote the paper. Y.M. and W.Y. did the statistical analysis, supervised by X.Z. All authors contributed to the data interpretation, revised each draft for important intellectual content, and read and approved the final manuscript.

\section{ADDITIONAL INFORMATION}

Supplementary Information is available for this paper at https://doi.org/10.1038/ s41416-018-0314-4.

Competing interests: The authors declare no competing interests.

Availability of data and materials: The data sets generated during and/or analysed during the current study are available for non-commercial use from the corresponding author on reasonable request.

Ethical approval/consent to participate: The study protocol was approved by the institutional review board of the Brigham and Women's Hospital and the Human Subjects Committee Review Board of the Harvard T. H. Chan School of Public Health.

Funding: No external funding was used for conducting this study.

Note: This work is published under the standard license to publish agreement. After 12 months the work will become freely available and the license terms will switch to a Creative Commons Attribution 4.0 International (CC BY 4.0)

\section{REFERENCES}

1. Siegel, R., Naishadham, D. \& Jemal, A. Cancer statistics, 2012. CA Cancer J. Clin. 62, 10-29 (2012).

2. Torre, L. A. et al. Global cancer statistics, 2012. CA Cancer J. Clin. 65, 87-108 (2015).

3. Campbell, P. T. et al. Prospective study reveals associations between colorectal cancer and type 2 diabetes mellitus or insulin use in men. Gastroenterology 139, 1138-1146 (2010).

4. Cavicchia, P. P. et al. Racial disparities in colorectal cancer incidence by type 2 diabetes mellitus status. Cancer Causes Control 24, 277-285 (2013).

5. Flood, A., Strayer, L., Schairer, C. \& Schatzkin, A. Diabetes and risk of incident colorectal cancer in a prospective cohort of women. Cancer Causes Control 21, 1277-1284 (2010).

6. He, J. et al. The association of diabetes with colorectal cancer risk: the multiethnic cohort. Br. J. Cancer 103, 120-126 (2010).

7. Hu, F. B. et al. Prospective study of adult onset diabetes mellitus (type 2) and risk of colorectal cancer in women. J. Natl. Cancer Inst. 91, 542-547 (1999).

8. Larsson, S. C., Giovannucci, E. \& Wolk, A. Diabetes and colorectal cancer incidence in the cohort of Swedish men. Diabetes Care 28, 1805-1807 (2005).

9. Larsson, S. C., Orsini, N. \& Wolk, A. Diabetes mellitus and risk of colorectal cancer: a meta-analysis. J. Natl. Cancer Inst. 97, 1679-1687 (2005).

10. Nilsen, T. I. \& Vatten, L. J. Prospective study of colorectal cancer risk and physical activity, diabetes, blood glucose and BMI: exploring the hyperinsulinaemia hypothesis. Br. J. Cancer 84, 417-422 (2001).

11. Peeters, P. J., Bazelier, M. T., Leufkens, H. G., de Vries, F. \& De Bruin, M. L. The risk of colorectal cancer in patients with type 2 diabetes: associations with treatment stage and obesity. Diabetes Care 38, 495-502 (2015).

12. Sun, L. \& Yu, S. Diabetes mellitus is an independent risk factor for colorectal cancer. Dig. Dis. Sci. 57, 1586-1597 (2012).

13. Yang, Y. X., Hennessy, S. \& Lewis, J. D. Type 2 diabetes mellitus and the risk of colorectal cancer. Clin. Gastroenterol. Hepatol. 3, 587-594 (2005). 
14. Seow, A., Yuan, J. M., Koh, W. P., Lee, H. P. \& Yu, M. C. Diabetes mellitus and risk of colorectal cancer in the Singapore Chinese Health Study. J. Natl. Cancer Inst. 98, 135-138 (2006).

15. Lee, M. S. et al. Type 2 diabetes increases and metformin reduces total, colorectal, liver and pancreatic cancer incidences in Taiwanese: a representative population prospective cohort study of 800,000 individuals. BMC Cancer 11, 20 (2011).

16. Limburg, P. J. et al. Diabetes mellitus and subsite-specific colorectal cancer risks in the lowa Women's Health Study. Cancer Epidemiol. Biomark. Prev. 14, 133-137 (2005).

17. Coughlin, S. S., Calle, E. E., Teras, L. R., Petrelli, J. \& Thun, M. J. Diabetes mellitus as a predictor of cancer mortality in a large cohort of US adults. Am. J. Epidemiol. 159, 1160-1167 (2004).

18. Inoue, M. et al. Diabetes mellitus and the risk of cancer: results from a large-scale population-based cohort study in Japan. Arch. Intern. Med. 166, 1871-1877 (2006).

19. de Kort, S. et al. Diabetes mellitus type 2 and subsite-specific colorectal cancer risk in men and women: results from the Netherlands Cohort Study on diet and cancer. Eur. J. Gastroenterol. Hepatol. 28, 896-903 (2016).

20. Limburg, P. J. et al. Clinically confirmed type 2 diabetes mellitus and colorectal cancer risk: a population-based, retrospective cohort study. Am. J. Gastroenterol. 101, 1872-1879 (2006).

21. Kuriki, K., Hirose, K. \& Tajima, K. Diabetes and cancer risk for all and specific sites among Japanese men and women. Eur. J. Cancer Prev. 16, 83-89 (2007).

22. Ren, $\mathrm{X}$. et al. Type 2 diabetes mellitus associated with increased risk for colorectal cancer: evidence from an international ecological study and population-based risk analysis in China. Public Health 123, 540-544 (2009).

23. Tabak, A. G. et al. Trajectories of glycaemia, insulin sensitivity, and insulin secretion before diagnosis of type 2 diabetes: an analysis from the Whitehall II study. Lancet 373, 2215-2221 (2009).

24. Giovannucci, E. Insulin and colon cancer. Cancer Causes Control 6, 164-179 (1995).

25. Vigneri, P., Frasca, F., Sciacca, L., Pandini, G. \& Vigneri, R. Diabetes and cancer. Endocr. Relat. Cancer 16, 1103-1123 (2009).

26. Luo, W., Cao, Y., Liao, C. \& Gao, F. Diabetes mellitus and the incidence and mortality of colorectal cancer: a meta-analysis of 24 cohort studies. Colorectal Dis. 14, 1307-1312 (2012).

27. Guraya, S. Y. Association of type 2 diabetes mellitus and the risk of colorectal cancer: a meta-analysis and systematic review. World J. Gastroenterol. 21, 6026-6031 (2015).

28. Guraya, S. Y. \& Eltinay, O. E. Higher prevalence in young population and rightward shift of colorectal carcinoma. Saudi. Med. J. 27, 1391-1393 (2006).

29. Tsilidis, K. K., Kasimis, J. C., Lopez, D. S., Ntzani, E. E. \& loannidis, J. P. Type 2 diabetes and cancer: umbrella review of meta-analyses of observational studies. BMJ 350, g7607 (2015).

30. Clendenen, T. V. et al. Postmenopausal levels of endogenous sex hormones and risk of colorectal cancer. Cancer Epidemiol. Biomark. Prev. 18, 275-281 (2009).

31. Falk, R. T. et al. Estrogen metabolites are not associated with colorectal cancer risk in postmenopausal women. Cancer Epidemiol. Biomark. Prev. 24, 1419-1422 (2015).

32. Gunter, M. J. et al. Insulin, insulin-like growth factor-l, endogenous estradiol, and risk of colorectal cancer in postmenopausal women. Cancer Res. 68, 329-337 (2008).

33. Lin, J. H. et al. Association between sex hormones and colorectal cancer risk in men and women. Clin. Gastroenterol. Hepatol. 11, 419-424 e411 (2013).

34. Murphy, N. et al. A prospective evaluation of endogenous sex hormone levels and colorectal cancer risk in postmenopausal women. J. Natl. Cancer. Inst. 107; https://doi.org/10.1093/jnci/djv210 (2015).

35. Shin, C. M. et al. Association among obesity, metabolic health, and the risk for colorectal cancer in the general population in Korea using the National Health Insurance Service-National Sample Cohort. Dis. Colon Rectum 60, 1192-1200 (2017).

36. Colditz, G. A. et al. Validation of questionnaire information on risk factors and disease outcomes in a prospective cohort study of women. Am. J. Epidemiol. 123, 894-900 (1986)

37. Simon, T. G. et al. Diabetes, metabolic comorbidities, and risk of hepatocellular carcinoma: results from two prospective cohort studies. Hepatology 67, 1797-1806 (2018)

38. Cornelis, M. C. et al. Joint effects of common genetic variants on the risk for type 2 diabetes in U.S. men and women of European ancestry. Ann. Intern. Med. 150, 541-550 (2009)
39. Manson, J. E. et al. Physical activity and incidence of non-insulin-dependent diabetes mellitus in women. Lancet 338, 774-778 (1991).

40. Classification and diagnosis of diabetes mellitus and other categories of glucose intolerance. National Diabetes Data Group. Diabetes 28, 1039-1057 (1979).

41. Hu, F. B. et al. Physical activity and television watching in relation to risk for type 2 diabetes mellitus in men. Arch. Intern. Med. 161, 1542-1548 (2001).

42. Willett, W. C. et al. Reproducibility and validity of a semiquantitative food frequency questionnaire. Am. J. Epidemiol. 122, 51-65 (1985).

43. Wu, K., Willett, W. C., Fuchs, C. S., Colditz, G. A. \& Giovannucci, E. L. Calcium intake and risk of colon cancer in women and men. J. Natl. Cancer Inst. 94, 437-446 (2002).

44. Rimm, E. B. et al. Reproducibility and validity of an expanded self-administered semiquantitative food frequency questionnaire among male health professionals. Am. J. Epidemiol. 135, 1114-1126 (1992). discussion 1127-1136.

45. Salvini, S. et al. Food-based validation of a dietary questionnaire: the effects of week-to-week variation in food consumption. Int. J. Epidemiol. 18, 858-867 (1989).

46. Feskanich, D. et al. Reproducibility and validity of food intake measurements from a semiquantitative food frequency questionnaire. J. Am. Diet. Assoc. 93, 790-796 (1993).

47. Willett, W. C. et al. Dietary fat and fiber in relation to risk of breast cancer. An 8year follow-up. JAMA 268, 2037-2044 (1992).

48. Cochran, W. G. The combination of estimates from different experiments. Biometrics 10, 101-129 (1954)

49. Facchini, F., Chen, Y. D. \& Reaven, G. M. Light-to-moderate alcohol intake is associated with enhanced insulin sensitivity. Diabetes Care 17, 115-119 (1994).

50. Kiechl, S. et al. Insulin sensitivity and regular alcohol consumption: large, prospective, cross sectional population study (Bruneck study). BMJ 313, 1040-1044 (1996).

51. Hamasaki, H. et al. Daily physical activity assessed by a triaxial accelerometer is beneficially associated with waist circumference, serum triglycerides, and insulin resistance in Japanese patients with prediabetes or untreated early type 2 diabetes. J Diabetes Res. 2015, 526201 (2015).

52. Cohen, F. J., Neslusan, C. A., Conklin, J. E. \& Song, X. Recent antihyperglycemic prescribing trends for US privately insured patients with type 2 diabetes. Diabetes Care 26, 1847-1851 (2003).

53. Hoerger, T. J., Segel, J. E., Gregg, E. W. \& Saaddine, J. B. Is glycemic control improving in U.S. adults? Diabetes Care 31, 81-86 (2008).

54. Rondini, E. A., Harvey, A. E., Steibel, J. P., Hursting, S. D. \& Fenton, J. I. Energy balance modulates colon tumor growth: interactive roles of insulin and estrogen. Mol. Carcinog. 50, 370-382 (2011).

55. Geer, E. B. \& Shen, W. Gender differences in insulin resistance, body composition, and energy balance. Gend. Med. 6(Suppl 1), 60-75 (2009).

56. Chlebowski, R. T. et al. Estrogen plus progestin and colorectal cancer in postmenopausal women. N. Engl. J. Med. 350, 991-1004 (2004).

57. Sainz, J. et al. Effect of type 2 diabetes predisposing genetic variants on colorectal cancer risk. J. Clin. Endocrinol. Metab. 97, E845-E851 (2012).

58. Kramer, H. U., Schottker, B., Raum, E. \& Brenner, H. Type 2 diabetes mellitus and colorectal cancer: meta-analysis on sex-specific differences. Eur. J. Cancer 48, 1269-1282 (2012).

59. Bell, R. A. et al. Associations between alcohol consumption and insulin sensitivity and cardiovascular disease risk factors: the Insulin Resistance and Atherosclerosis Study. Diabetes Care 23, 1630-1636 (2000).

60. Klarich, D. S., Brasser, S. M. \& Hong, M. Y. Moderate alcohol consumption and colorectal cancer risk. Alcohol. Clin. Exp. Res. 39, 1280-1291 (2015).

61. Kontou, N. et al. Alcohol consumption and colorectal cancer in a Mediterranean population: a case-control study. Dis. Colon Rectum 55, 703-710 (2012).

62. Park, J. Y. et al. Baseline alcohol consumption, type of alcoholic beverage and risk of colorectal cancer in the European Prospective Investigation into Cancer and Nutrition-Norfolk study. Cancer Epidemiol. 33, 347-354 (2009).

63. $\mathrm{Ma}$, J. et al. A prospective study of plasma C-peptide and colorectal cancer risk in men. J. Natl. Cancer Inst. 96, 546-553 (2004).

64. Yin, S., Bai, H. \& Jing, D. Insulin therapy and colorectal cancer risk among type 2 diabetes mellitus patients: a systemic review and meta-analysis. Diagn. Pathol. 9, 91 (2014).

65. Liu, F. et al. Metformin therapy and risk of colorectal adenomas and colorectal cancer in type 2 diabetes mellitus patients: a systematic review and metaanalysis. Oncotarget 8, 16017-16026 (2017).

66. Johnson, J. A., Bowker, S. L., Richardson, K. \& Marra, C. A. Time-varying incidence of cancer after the onset of type 2 diabetes: evidence of potential detection bias. Diabetologia 54, 2263-2271 (2011). 